Check for updates

Cite this: Chem. Sci., 2019, 10, 3025

๑ All publication charges for this article have been paid for by the Royal Society of Chemistry

Received 30th November 2018 Accepted 22nd January 2019

DOI: $10.1039 / \mathrm{c} 8 \mathrm{sc} 05358 \mathrm{~h}$

rsc.li/chemical-science

\title{
Durable liquid-crystalline vitrimer actuators $\uparrow$
}

\author{
Qiaomei Chen, (D) ${ }^{a}$ Yongsan $\mathrm{Li}^{\mathrm{a}}{ }^{\mathrm{Y}}$ Yang Yang, (D) ${ }^{\mathrm{a}}$ Yanshuang $\mathrm{Xu},{ }^{\mathrm{b}}$ Xiaojie Qian, ${ }^{\mathrm{a}}$ \\ Yen Wei ${ }^{\text {ac }}$ and Yan $\mathrm{Ji}^{\star a}$
}

Vitrimer-based liquid-crystalline elastomers (LCEs) exhibit great advantages over the traditional LCEs due to their inherent processability to realize monodomain alignment and construction of LCE actuators with complex 3D structures in a robust way. Though exciting progress has been made, how to achieve a proper balance between processability and actuation durability/stability remains a big challenge. Here, we report a strategy to mitigate the conflict between processability and actuation stability by reducing the catalyst content in an epoxy/acid LCE vitrimer system. With a relatively low catalyst content (0.25 mol\% to carboxyl group), monodomain LCEs with large actuation strain ( 95\%) and excellent actuation stability (the actuation strain is completely maintained after 100 heating-cooling cycles and more than $90 \%$ of the initial strain is retained even after 500 cycles) could be easily prepared. Moreover, the monodomain LCEs can still be readily realigned or directly reconfigured into complex reversible 3D actuators.

\section{Introduction}

Thanks to their unique characteristics like light weight, low cost and flexible actuation, polymer soft actuators are considered to be essential for advanced high-tech fields such as micromechanical systems, artificial muscles and soft robotics. ${ }^{1-5}$ Recently, they have attracted massive attention from both science and industry communities. Among the many reported polymer soft actuators, liquid-crystalline elastomers (LCEs) stand out due to their unique large reversible high-speed actuations (resulting from the isotropy to anisotropy transition). ${ }^{6}$ To realize free-standing actuation, macroscopic alignment of the liquid-crystal order (monodomain LCEs) is necessary. Traditionally, aligning before complete curing is inevitable as LCEs are constructed by covalently cross-linked networks which cannot be reprocessed once synthesized. Varieties of methods and techniques were developed on this basis, including the twostep crosslinking method, ${ }^{7,8}$ in situ photoalignment, ${ }^{9,10}$ external fields, ${ }^{11-13}$ soft lithography, ${ }^{14,15}$ inkjet printing ${ }^{16,17}$ and microfluidics. ${ }^{18,19}$ Although much progress has been achieved, still they suffer from some inherent disadvantages, especially the

\footnotetext{
${ }^{a}$ MOE Key Laboratory of Bioorganic Phosphorus Chemistry \& Chemical Biology, Department of Chemistry, Tsinghua University, Beijing 100084, China. E-mail: jiyan@mail.tsinghua.edu.cn

${ }^{b}$ Guangxi Key Laboratory of Petrochemical Resource Processing and Process Intensification Technology, School of Chemistry and Chemical Engineering, Guangxi University, Nanning 530004, China

'Department of Chemistry, Center for Nanotechnology and Institute of Biomedical Technology, Chung-Yuan Christian University, Chung-Li 32023, Taiwan

$\dagger$ Electronic supplementary information (ESI) available. See DOI: $10.1039 / \mathrm{c} 8 \mathrm{sc} 05358 \mathrm{~h}$
}

difficulty to obtain complex 3D shapes, restricting the practical applications of LCE actuators.

To break through the aforementioned bottleneck, our group and other researchers contributed to putting forward a brand new strategy by synthesizing LCEs with exchangeable links especially vitrimers. ${ }^{20-29}$ Vitrimers are covalently crosslinked networks that are able to change their topology due to the exchange reactions under certain external stimuli. The formation of new covalent bonds and breaking of old ones occur at the same time, resulting in the constant number of chemical bonds and cross-linking density during the exchange reactions. ${ }^{30,31}$ This strategy gives a new scope for monodomain alignment, overcoming the challenges of limited sample size, unreliable processes and sophisticated 3D structure construction. To date, most of the LCEs with exchangeable links were prepared based on transesterification ${ }^{20-26}$ and disulfide ${ }^{27}$ and allyl sulfide groups. ${ }^{28,29}$ LCE actuators prepared by lighttriggered allyl sulfide exchange reaction are known to possess excellent thermal stability. However, once the photoinitiators were consumed during the alignment, the only way to regain their processability is to swell the LCEs in solutions containing photoinitiators, which is time-consuming and environmentally unfriendly. For networks formed with ester and disulfide groups, the exchange reactions could be triggered by high temperature. On one hand, they contribute to the monodomain alignment. On the other hand, when the actuators work at temperatures above the topology-freezing transition temperature $\left(T_{\mathrm{v}}\right)$, the exchange reactions would be activated and impair the monodomain alignment, leading to actuation failure. As is known, macroscopic actuations of LCEs are resulted from the liquid-crystalline isotropic transition $\left(T_{\mathrm{i}}\right)$; that is to say, $T_{\mathrm{v}}$ should be higher than $T_{\mathrm{i}}$ as much as possible (except for 
azobenzene-based LCEs) to improve the durability of the LCE actuators. Moreover, to enable processability with suitable time and temperature (long time and high temperature would affect the functions of the materials), a balance between stability and processability is needed. To date, it remains very challenging to prepare vitrimer-based LCE actuators with excellent actuation stability as well as processability, which greatly restricts their potential and practical applications.

Here, we report a strategy to alleviate the strife between the actuation stability and the processability of vitrimer-based LCEs by adjusting the catalyst amount. It is widely acknowledged that catalysts are vital in controlling reaction rates, which remains true for catalyst activated exchange reactions in vitrimers. Leibler's group developed an epoxy/acid vitrimer system by introducing a transesterification catalyst into the system cured by fatty acid/anhydride. At temperatures higher than $T_{\mathrm{v}}$, ester groups are capable of exchanging with hydroxyl groups to generate new ester groups and new hydroxyl groups, resulting in processability of the crosslinked network. ${ }^{32}$ This group has justified that the $T_{\mathrm{v}}$ value of the epoxy/acid vitrimer networks can be tuned in a controllable way by changing the content and nature of the catalyst. ${ }^{33}$ In this work, we manage to maintain the monodomain alignment (with large actuation strain up to 95\%) after 100 heating-cooling cycles $\left(100{ }^{\circ} \mathrm{C}\right.$ to $30{ }^{\circ} \mathrm{C}$, about $37{ }^{\circ} \mathrm{C}$ above its $T_{\mathrm{i}}$ ) with a relatively low catalyst content of $0.25 \mathrm{~mol} \%$ to the carboxyl group. And more than $90 \%$ of the initial strain could be retained even after 500 cycles. The aligned sample can be utilized to do mechanical works, such as lifting a weight about 676 times its weight with a strain of $82 \%$. Owing to the high temperature transesterification reaction, the monodomain alignment can be fully erased and realigned, reaching nearly the same actuation strain as that of the first alignment. Moreover, the obtained flat monodomain sample can be directly reconfigured into 3D structures at high temperature and retain some of the orientations at the same time, resulting in reversible actuators with complex 3D structures.

\section{Results and discussion}

\section{Preparation and characterization of the vitrimer-based LCE}

As shown in Fig. 1a, the vitrimer-based LCE was synthesized via reacting the diglycidyl ether of $4,4^{\prime}$-dihydroxy- $\alpha$-methylstilbene (DGE-DHMS) and sebacic acid under the catalysis of triazabicyclodecene (TBD), where the stoichiometry of carboxyl and epoxy groups was $1: 1$, contributing to the formation of $\beta$ hydroxyl-esters and the transesterification reaction. We chose DGE-DHMS as the liquid-crystalline monomer instead of the diglycidyl ether of 4,4'-dihydroxybiphenol (DGE-DHBP) in our previous work $^{\mathbf{2 0}}$ due to its lower liquid-crystalline isotropic transition $T_{\mathrm{i}}$ and longer mesogenic groups, resulting in a LCE vitrimer with lower $T_{\mathrm{i}}$ and larger actuation strain..$^{34}$

The effect of the catalyst content on the network plasticity was first investigated. Networks were synthesized with different contents of TBD with $5 \%, 2.5 \%, 1 \%$ and $0.25 \%$ mole ratios to the carboxyl group. Shear stress relaxation experiments were performed in the linear viscoelastic regime (the applied strain $\gamma$ $=1 \%$ of the samples with different catalyst contents at different temperatures between the isotropic transition temperature $T_{\mathrm{i}}$ and the decomposition temperature $T_{\mathrm{d}}$ (the onset of decomposition for the sample with a catalyst content of $0.25 \mathrm{~mol} \%$ is about $309{ }^{\circ} \mathrm{C}$ under an air atmosphere; Fig. S4 $\dagger$ ) until the samples had relaxed to below $1 / e$ of the initial stress relaxation modulus (the time needed is defined as relaxation times $\left.\tau^{*}\right)$. As shown in Fig. S5, $\uparrow$ the higher the temperature, the faster the stress relaxation for all the samples, i.e., the shorter the relaxation times $\tau^{*}$. The shear stress relaxation curves (Fig. 1b) obtained at the same temperature $\left(200^{\circ} \mathrm{C}\right)$ for the four different samples reveal that $\tau^{*}$ decreases with increasing catalyst contents. Moreover, modulus-temperature curves and swelling experiments of the four samples show that both the average plateau modulus and crosslinking density increase slightly with decreasing catalyst content. Xie's group has reported that larger crosslinking density leads to slower stress relaxation in networks with dynamic covalent bonds ${ }^{35}$ revealing that the higher crosslinking density of the sample with lower catalyst content also has some contribution to the lower stress relaxation.

Relaxation times $\tau^{*}$ follow an Arrhenius law at temperature above $T_{\mathrm{v}} . T_{\mathrm{v}}$ values of the four samples with different catalyst contents were determined using the Maxwell equation according the method previously reported (the details can be found in the ESI $\dagger$ ). ${ }^{36}$ As shown in Fig. 1c, the values of $T_{\mathrm{v}}$ for the samples with catalyst contents of $5 \%, 2.5 \%, 1 \%$ and $0.25 \%$ are $67{ }^{\circ} \mathrm{C}$, $87{ }^{\circ} \mathrm{C}, 103{ }^{\circ} \mathrm{C}$ and $132{ }^{\circ} \mathrm{C}$, revealing that $T_{\mathrm{v}}$ increases with decreasing catalyst content.

\section{Monodomain LCE preparation}

The transesterification reaction triggered by high temperatures can rearrange the network under external forces to obtain the monodomain alignment, but it can also diminish the alignment without external forces at temperatures above $T_{\mathrm{v}}$. It has been proved as mentioned above that $T_{\mathrm{v}}$ increases with decreasing catalyst content. To improve the actuation stability of the monodomain LCE, it is an effective way to reduce the catalyst content to improve $T_{\mathrm{v}}$. We chose the catalyst content of $0.25 \%$ to prepare the monodomain LCE actuators in this paper. Monodomain alignment is realized by stretching the sample at temperature above $T_{\mathrm{v}}\left(180^{\circ} \mathrm{C}\right)$. In the LCE vitrimer (xLCE) our group previously reported, ${ }^{19}$ the transesterification reaction is so fast that post-stretching during the cooling procedure is essential to maintain the alignment. Thanks to the lower catalyst content in the LCE vitrimer reported here, the speed of the transesterification reaction drops significantly. Monodomain alignment can be obtained by just simply fixing the sample on the surface of a glass slide with the aimed stretching strain and then heating it without post-stretching during the cooling procedure, which simplifies the alignment process and improves the success rate. As shown in Fig. 2a, when the sample was heated at $180{ }^{\circ} \mathrm{C}$ under external force, the polymer chains were first aligned and the transesterification is activated meanwhile to relax the stress, leading to a new aligned topology. We investigated the effect of the stretching strain and heating time on the actuation strain by the variable-controlling 
a
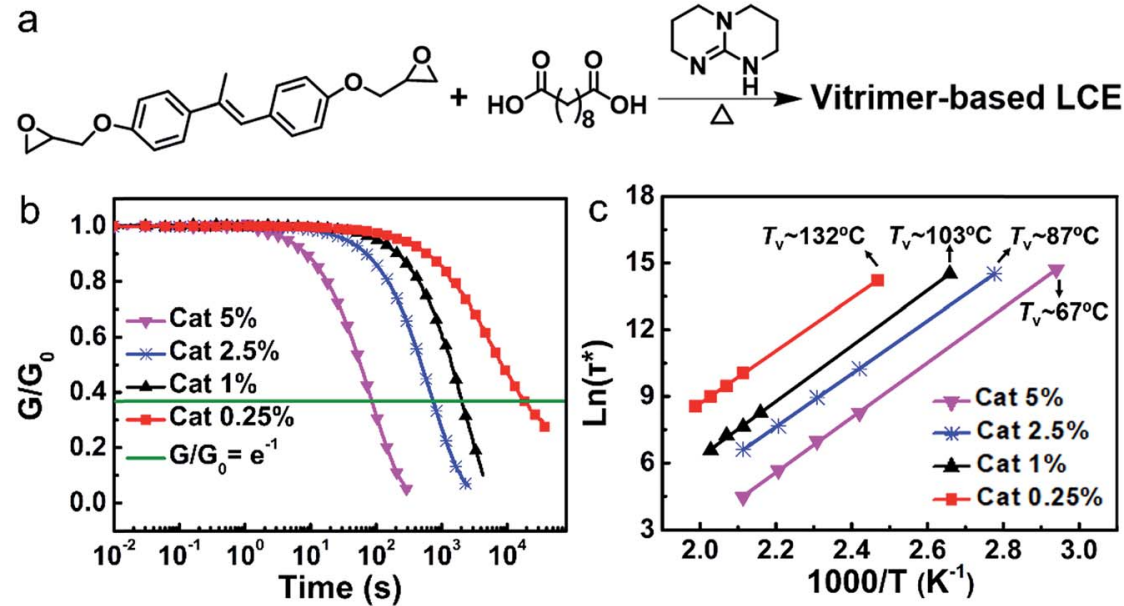

Fig. 1 Synthesis of the vitrimer-based LCE and the effect of catalyst content on the network plasticity. (a) The synthesis of the vitrimer-based LCE. (b) Normalized shear stress relaxation of the samples with different catalyst contents at $200^{\circ} \mathrm{C}$ (constant $1 \%$ strain applied). (c) Arrhenius plot of the measured relaxation times for the samples with different catalyst contents.

approach, where the heating time (30 min) and stretching strain $(50 \%)$ are two constants, respectively. As shown in Fig. $2 \mathrm{~b}$, the actuation strain increased first with the stretching strain, but it reached a plateau when the stretching strain was above $50 \%$, meaning that a higher stretching strain above 50\% makes no significant contribution to the monodomain alignment in the vitrimer-based LCE reported here. The heating time has a similar trend of influence on the actuation strain, which approaches a constant at heating time above $30 \mathrm{~min}$. We can also find that the heating time shows a more significant impact on the actuation strain than the stretching strain. Based on the above investigations, monodomain LCEs in the following were prepared via heating at $180^{\circ} \mathrm{C}$ for $30 \mathrm{~min}$ with a constant strain of $50 \%$. The spontaneous actuation strain reaches as high as 90-95\% when the aligned film transforms from an isotropic to anisotropic phase (Fig. 2c). The alignment of the monodomain
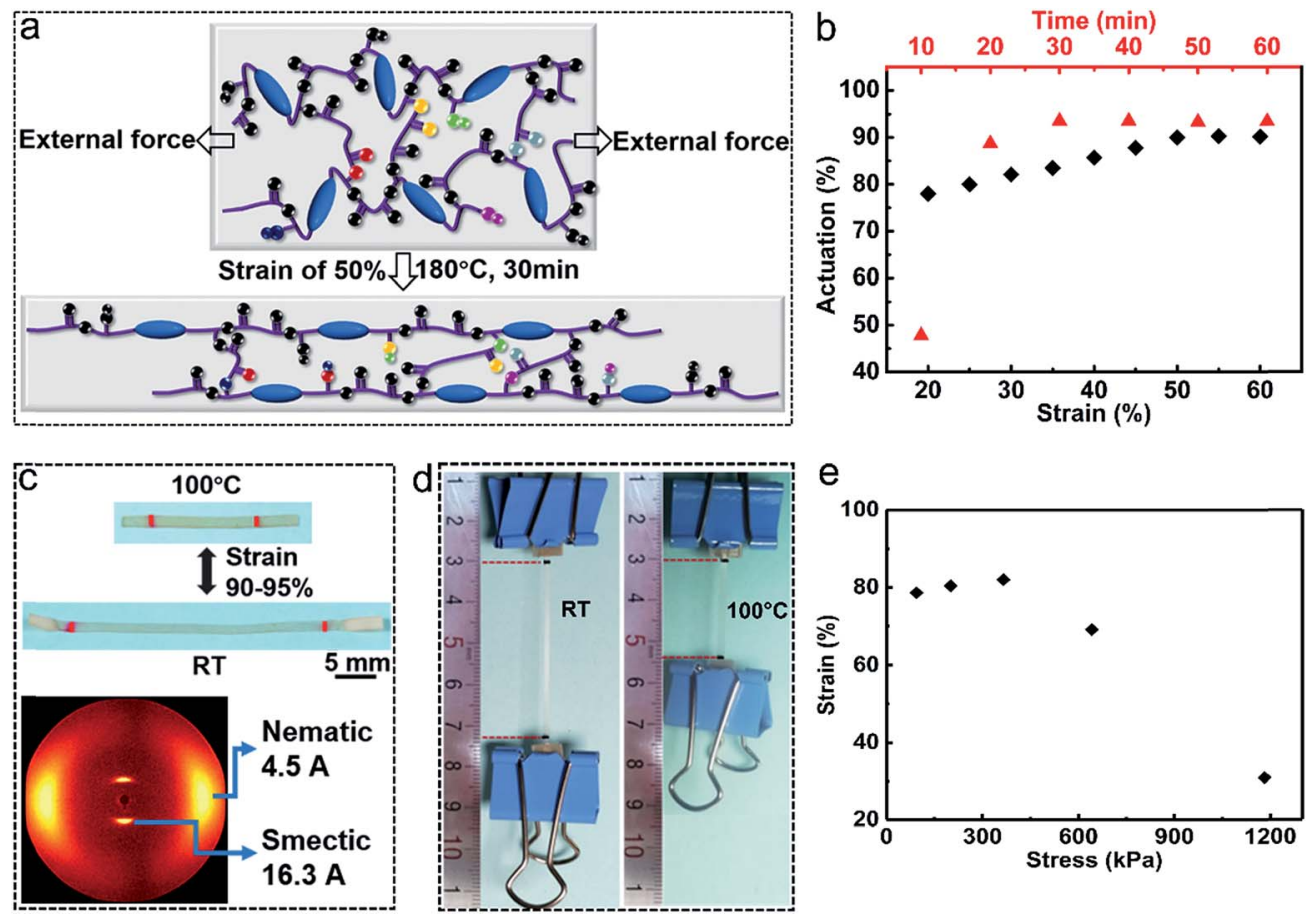

Fig. 2 Preparation of the monodomain LCE. (a) An illustration of monodomain alignment, where the blue ellipsoids represent mesogens, the larger pellets represent oxygen atoms and the smaller pellets represent hydrogen atoms. (b) Strain/time-actuation scattering diagram of the vitrimer-based LCEs. (c) Thermal actuation of the monodomain LCE and the corresponding X-ray diffraction image. (d) A demonstration of the monodomain LCE doing mechanical work, where the weight of the metal clip is about $4.93 \mathrm{~g}$ (about 675 times heavier than the sample) and the induced stress is about $366 \mathrm{kPa}$. (e) Stress-strain scattering diagram of the vitrimer-based LCE for mechanical works. 

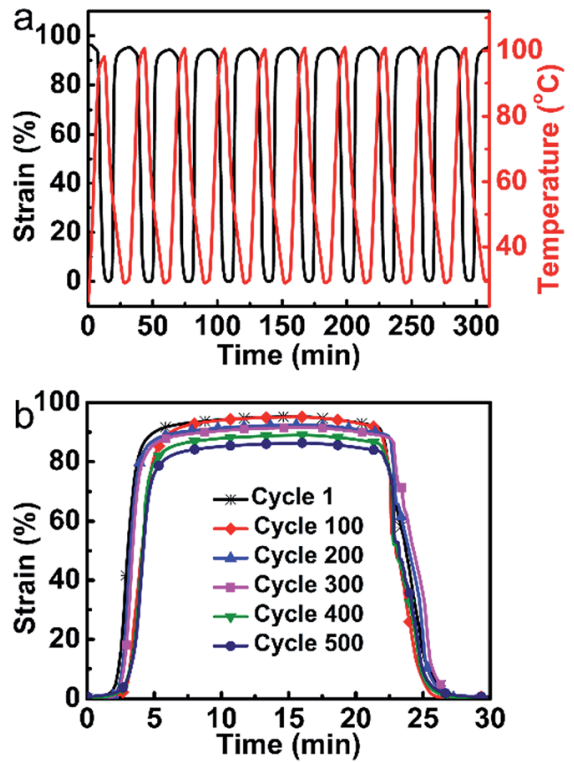

Fig. 3 Repeated actuation stability investigations of the monodomain LCE. (a) The first 10 cycles. (b) Actuation strain changes of every 100 cycles.

LCE was also confirmed by 2D X-ray diffraction, which showed a well-aligned smectic-A order with a high orientational (nematic) order parameter $f=0.70$ (Fig. 2c; the details can be found in the ESI $\dagger$ ).

Owing to the relatively excellent mechanical strength of smectic LCEs and large actuation strain of the vitrimer-based LCE reported here, the monodomain LCE can be applied for mechanical works. As a demonstration, the aligned monodomain sample (about $7.30 \mathrm{mg}$ ) was loaded with a metal clip of about $4.93 \mathrm{~g}$. When heated to temperature above $T_{\mathrm{i}}\left(100^{\circ} \mathrm{C}\right)$, the sample contracted quickly and lifted the metal clip up for about $19.3 \mathrm{~mm}$ (the strain of the sample at $T_{\mathrm{i}}$ is about $82 \%$ ) (Fig. 2d). Fig. 2e shows the strain of the monodomain sample loaded with different weights. The results show that the strain first increases with increasing weight and then decreases, which reveals that the weight contributes to the mesogen alignment to some extent but will ultimately hinder the shrinkage of the sample with higher weight.

\section{Actuation stability}

Compared to the previously reported LCE vitrimers with heattriggered actuations, the vitrimer-based LCE reported here with a low catalyst content has distinct advantages in terms of stability. Fig. 3a shows the first 10 cycles of heating-cooling $\left(100{ }^{\circ} \mathrm{C}\right.$ to $30^{\circ} \mathrm{C}$, about $37{ }^{\circ} \mathrm{C}$ above $T_{\mathrm{i}}$; the value can be obtained from Fig. S3 ${ }^{\dagger}$ ) of the monodomain sample, which reveals an excellent stability with an actuation strain of about $95 \%$. As shown in Fig. 3b, the actuation strain is totally retained after 100 cycles. Even after 500 cycles, the actuation strain (about $86 \%$ ) only decreased less than $10 \%$ of the initial strain. Control experiments with higher catalyst contents have also been conducted. For the sample with $5 \mathrm{~mol} \%$ catalyst content, the transesterification rate is too high to maintain the orientation order at temperature above $T_{\mathrm{i}}$; for the sample with $1 \mathrm{~mol} \%$ catalyst content, the actuation performance has obviously declined (decreased about $4 \%$ of the initial strain) only after 50 cycles (Fig. S9†). All the above results justify the excellent stability of the monodomain LCE reported here for actuation.
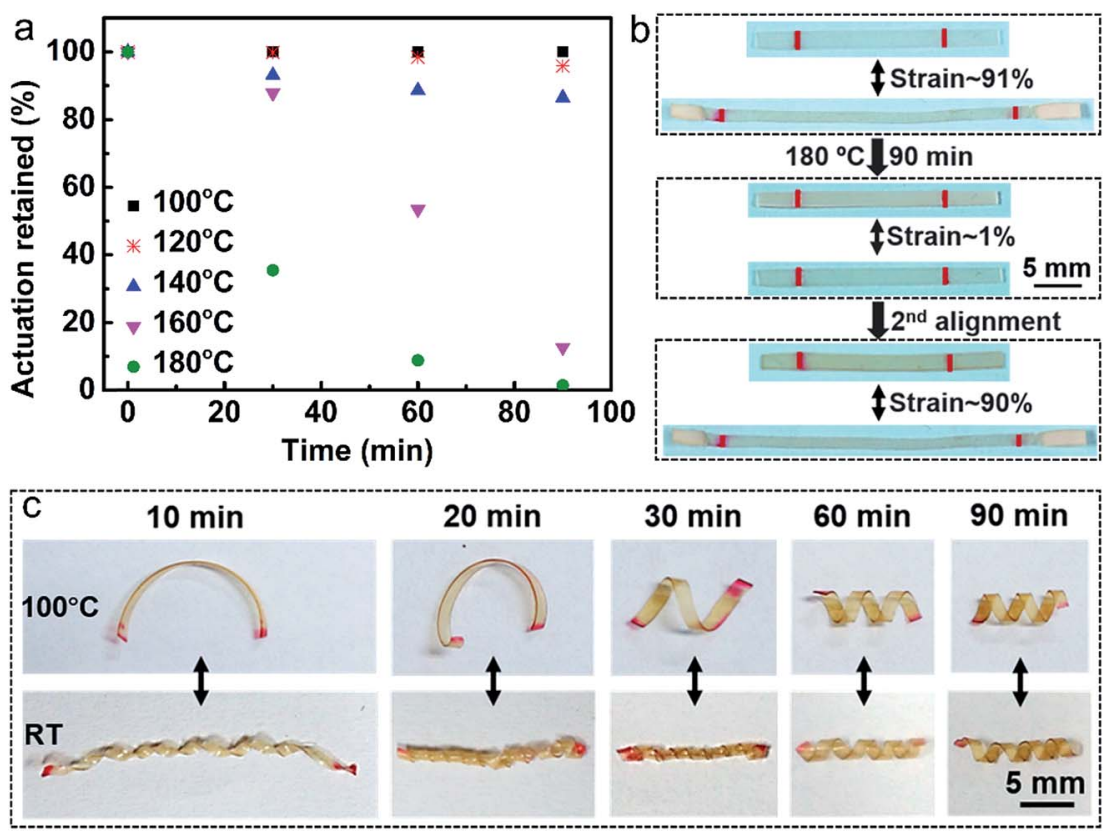

Fig. 4 Reprogramming of the monodomain LCE. (a) The actuation changed over heating time at different temperatures. (b) Diminishing and realignment of the orientation order. (c) Various actuators with 3D structures prepared by directly reconfiguring the flat monodomain sample at high temperature for different heating times. 


\section{Reprogramming of the monodomain LCE}

The small amount of the catalyst also enables the monodomain LCE to be reprogrammed. At elevated temperature, transesterification reaction is activated to diminish the alignment, resulting in a decrease in the orientation order with time. We investigated the actuation strain of the monodomain samples versus time after heating at $100{ }^{\circ} \mathrm{C}, 120{ }^{\circ} \mathrm{C}, 140{ }^{\circ} \mathrm{C}, 160{ }^{\circ} \mathrm{C}$ and $180{ }^{\circ} \mathrm{C}$, respectively (Fig. 4a). As expected, the higher the temperature, the faster the decrease of the orientation order. For example, the sample heated at $100{ }^{\circ} \mathrm{C}$ for $90 \mathrm{~min}$ could retain all its initial actuation strain, but after heated at $180{ }^{\circ} \mathrm{C}$ for $90 \mathrm{~min}$, the sample nearly lost all the orientation order. Owing to the catalyst and transesterification reaction, the orientation order disappeared sample can be realigned and obtain nearly the same actuation strain (Fig. 4b). Thus when the actuation of the actuators cannot reach the standard during long service time, they can be simply realigned without the preparation of new materials, which is time and energy saving.

Previously, it has been verified that the additional stretching of chains can provide an extra tension on the crosslinking bonds and reduce the effective activation energy, resulting in the lower topology-freezing transition temperature $T_{\mathrm{v}}$ than the free-standing sample. ${ }^{37,38}$ In accordance with the above theory, we find that it indeed takes more time for the free-standing monodomain sample to lose all its orientation than to align it at the same temperature. Utilizing the transesterification reaction, we can further directly reconfigure the flat monodomain sample to obtain 3D structures and retain some of the orientations at the same time. Five strips of flat monodomain samples similar to that in Fig. 2c were first spirally winded on the surface of five fine rods to reconfigure them at $180{ }^{\circ} \mathrm{C}$ for $10 \mathrm{~min}, 20 \mathrm{~min}, 30 \mathrm{~min}, 60 \mathrm{~min}$ and $90 \mathrm{~min}$, respectively. As shown in Fig. 4c, on one hand, the shape changes of the reconfigured samples at temperature above $T_{\mathrm{i}}\left(100^{\circ} \mathrm{C}\right)$ become more and more obvious with time increasing, which nearly do not change after $60 \mathrm{~min}$, revealing that $60 \mathrm{~min}$ is enough for the sample to relax all the stress during the spiral winding. On the other hand, the monodomain alignment decreases with configuration time increasing. The above two aspects resulted in the reversible complex $3 \mathrm{D}$ actuators with both shape and length change at $T_{\mathrm{i}}$. Thus we can choose the suitable actuation performance by controlling the reconfiguration time.

\section{Conclusions}

LCEs are expected to play a substantial role as actuators and sensors in a variety of applications. Despite their high potential, proper LCEs are still not available for the easy mass production of 3D soft actuators. The vitrimer-based LCEs have attracted much attention of researchers for 3D soft actuators in recent years. Actuation stability and suitable processability are two key factors in vitrimer-based LCE actuators. The main point of this work is to seek a route to produce high performance LCEs with both good stability and processability. By reducing the catalyst content to a relatively low level, the topology-freezing transition temperature $T_{\mathrm{v}}$ increased (greatly improving the actuation stability) and the processability of vitrimers was still retained (enabling the monodomain alignment, realignment or reconfiguration of the vitrimer-based LCE). The strategy introduced here is not limited to the current system. Moreover, based on this strategy, other methods such as decreasing the density or increasing the distance of the exchangeable site could also improve the actuation stability of vitrimer-based LCE actuators. We hope that this work would accelerate the practical applications of LCE actuators in areas such as micromechanical systems, artificial muscles and soft robotics.

\section{Conflicts of interest}

There are no conflicts to declare.

\section{Acknowledgements}

This research was supported by the National Natural Science Foundation of China (no. 21674057 and 51722303).

\section{References}

1 Y. Bahramzadeh and M. Shahinpoor, Soft Robotics, 2014, 1, 38-52.

2 T. Mirfakhrai, J. D. W. Madden and R. H. Baughman, Mater. Today, 2007, 10, 30-38.

3 S. Taccola, F. Greco, E. Sinibaldi, A. Mondini, B. Mazzolai and V. Mattoli, Adv. Mater., 2015, 27, 1668-1675.

4 Y. Yu and T. Ikeda, Angew. Chem., Int. Ed., 2006, 45, 54165418.

5 C. Majidi, Soft Robotics, 2014, 1, 5-11.

6 C. Ohm, M. Brehmer and R. Zentel, Adv. Mater., 2010, 22, 3366-3387.

7 J. Küpfer and H. Finkelmam, Macromol. Chem. Phys., 1994, 195, 1353-1367.

8 L. Liu, M.-H. Liu, L.-L. Deng, B.-P. Lin and H. Yang, J. Am. Chem. Soc., 2017, 139, 11333-11336.

9 H. Zeng, O. M. Wani, P. Wasylczyk, R. Kaczmarek and A. Priimagi, Adv. Mater., 2017, 29, 1701814.

10 S.-k. Ahn, T. H. Ware, K. M. Lee, V. P. Tondiglia and T. J. White, Adv. Funct. Mater., 2016, 26, 5819-5826.

11 S. Schuhladen, F. Preller, R. Rix, S. Petsch, R. Zentel and H. Zappe, Adv. Mater., 2014, 26, 7247-7251.

12 T. H. Ware, M. E. McConney, J. J. Wie, V. P. Tondiglia and T. J. White, Science, 2015, 347, 982-984.

13 W. Lehmann, H. Skupin, C. Tolksdorf, E. Gebhard, R. Zentel, P. Krüger, M. Lösche and F. Kremer, Nature, 2001, 40, 447450.

14 A. Buguin, M.-H. Li, P. Silberzan, B. Ladoux and P. Keller, J. Am. Chem. Soc., 2006, 128, 1088-1089.

15 H. Yang, G. Ye, X. Wang and P. Keller, Soft Matter, 2011, 7, 815-823.

16 C. L. van Oosten, C. W. M. Bastiaansen and D. J. Broer, Nat. Mater., 2009, 8, 677-682.

17 H. Minemawari, T. Yamada, H. Matsui, J. Tsutsumi, S. Haas, R. Chiba, R. Kumai and T. Hasegawa, Nature, 2011, 475, 364367. 
18 C. Ohm, N. Kapernaum, D. Nonnenmacher, F. Giesselmann, C. Serra and R. Zentel, J. Am. Chem. Soc., 2011, 133, 53055311.

19 C. Ohm, E.-K. Fleischmann, I. Kraus, C. Serra and R. Zentel, Adv. Funct. Mater., 2010, 20, 4314-4322.

20 Z. Pei, Y. Yang, Q. Chen, E. M. Terentjev, Y. Wei and Y. Ji, Nat. Mater., 2014, 13, 36-41.

21 X. Lu, H. Zhang, G. Fei, B. Yu, X. Tong, H. Xia and Y. Zhao, Adv. Mater., 2018, 30, e1706597.

22 T. Ube, K. Kawasaki and T. Ikeda, Adv. Mater., 2016, 28, 8212-8217.

23 X. Lu, S. Guo, X. Tong, H. Xia and Y. Zhao, Adv. Mater., 2017, 29, 1606467.

24 Y. Yang, Z. Pei, Z. Li, Y. Wei and Y. Ji, J. Am. Chem. Soc., 2016, 138, 2118-2121.

25 Q. Chen, Y. Wei and Y. Ji, Chin. Chem. Lett., 2017, 28, 21392142.

26 Y. Yang, X. Zhang, Y. Wei and Y. Ji, Acta Polym. Sin., 2017, 10, 1662-1667.

27 Y. Li, Y. Zhang, O. Rios, J. K. Keum and M. R. Kessler, RSC Adv., 2017, 7, 37248-37254.
28 X. Qian, Q. Chen, Y. Yang, Y. Xu, Z. Li, Z. Wang, Y. Wu, Y. Wei and Y. Ji, Adv. Mater., 2018, 30, 1801103.

29 M. K. McBride, M. Hendrikx, D. Liu, B. T. Worrell, D. J. Broer and C. N. Bowman, Adv. Mater., 2017, 29, 1606509.

30 W. Denissen, J. M. Winne and F. E. Du Prez, Chem. Sci., 2016, 7, 30-38.

31 W. Zou, J. Dong, Y. Luo, Q. Zhao and T. Xie, Adv. Mater., 2017, 29, 1606100.

32 D. Montarnal, M. Capelot, F. Tournilhac and L. Leibler, Science, 2011, 334, 965-968.

33 M. Capelot, M. M. Unterlass, F. Tournilhac and L. Leibler, ACS Macro Lett., 2012, 1, 789-792.

34 M. Giamberjni, E. Amendola and C. Carfagna, Mol. Cryst. Liq. Cryst., 1995, 266, 9-22.

35 Z. Fang, N. Zheng, Q. Zhao and T. Xie, ACS Appl. Mater. Interfaces, 2017, 9, 22077-22082.

36 J. P. Brutman, P. A. Delgado and M. A. Hillmyer, ACS Macro Lett., 2014, 3, 607-610.

37 Y. Yang, E. M. Terentjev, Y. Wei and Y. Ji, Nat. Commun., 2018, 9, 1906.

38 F. Meng, R. H. Pritchard and E. M. Terentjev, Macromolecules, 2016, 49, 2843-2852. 